Journal of Computer Science 6 (2): 217-223, 2010

ISSN 1549-3636

(C) 2010 Science Publications

\title{
Skin Images Segmentation
}

\author{
Ali El-Zaart \\ Department of Computer Science, College of Computer and Information Sciences, \\ King Saud University, Riyadh, Saudi Arabia
}

\begin{abstract}
Problem statement: Image segmentation is a fundamental step in many applications of image processing. Skin cancer has been the most common of all new cancers detected each year. At early stage detection of skin cancer, simple and economic treatment can cure it mostly. An accurate segmentation of skin images can help the diagnosis to define well the region of the cancer. The principal approach of segmentation is based on thresholding (classification) that is lied to the problem of the thresholds estimation. Approach: The objective of this study is to develop a method to segment the skin images based on a mixture of Beta distributions. We assume that the data in skin images can be modeled by a mixture of Beta distributions. We used an unsupervised learning technique with Beta distribution to estimate the statistical parameters of the data in skin image and then estimate the thresholds for segmentation. Results: The proposed method of skin images segmentation was implemented and tested on different skin images. We obtained very good results in comparing with the same techniques with Gamma distribution. Conclusion: The experiment showed that the proposed method obtained very good results but it requires more testing on different types of skin images.
\end{abstract}

Key words: Image segmentation, beta distribution, skin images, unsupervised learning technique

\section{INTRODUCTION}

Image segmentation is an important step in image analysis, pattern recognition, and computer vision. In radar images, for oil slicks detection, the segmentation is the main step for detecting the oil slick and defining its boundary (El Zaart et al., 2002). In mammography images, the segmentation is used to detect the region of the breast cancer (El Zaart et al., 2004; Ferrari et al., 2004). In Magnetic Resonance Images (MRI), segmentation of tissues is essential especially for a radiologist to be able to identify a disease, tumors, or any tissue (Mitsias et al., 2002). In skin images, the segmentation can detect the cancer regions (Xua et al., 1999; Ercal et al., 1993). In this study, we will work on the segmentation of skin images in order to define the boundaries of the skin regions. Many techniques exist for image segmentation based on different methods. There are four broad classes of segmentation methods, which are: classification-based methods, edge-based methods, region-based methods and, hybrid methods (Gonzalez and Woods, 2008). The principal approach of segmentation is based on thresholding that is related to the problem of the thresholds estimation. Iterative self-organizing data analysis technique is one of the thresholding methods in image segmentation. It is an unsupervised learning technique. Statistical approach is wieldy used in image processing in order to model the data of image. Gaussian and Gamma distributions have been used in this technique. Gaussian can only approximate a symmetric shape of histogram. Gamma distribution can only approximate a symmetric shape and a skewed to right shape of the histogram. However, the Beta distribution is more general than Gaussian and Gamma and it can approximate any shape of histogram as skewed to left, skewed to right and symmetric (El Zaart and Ziou, 2007). The algorithm developed here based on the technique of unsupervised learning using a mixture of Beta distributions. In the follows, we compare Beta with other distributions and give a definition of the Beta distribution.

Statistical distributions: A statistical distribution can be characterized by two measures: Skewness $(\beta 1)$ and kurtosis ( $\beta 2)$. We present a comparison among the following four distributions: Gaussian, Log-Normal, Gamma and Beta regarding those two measures. Gaussian distribution has a skewness of zero because it is symmetrical about the mean. The Log-Normal distribution is often skewed, with a slowly decreasing right tail. Both of the Beta and Gamma distributions satisfy the basic criteria of uni-modality and rightward skew, but the Gamma distribution differs from the Beta by having a shorter initial phase of low slope and a lower peak (El Zaart and Ziou, 2007). Beta distribution can be skewed to left as well but Gamma cannot have 
the shape skewed to left. This is the main advantage of Beta over other distributions.

As shown in Fig. 1, in the space of $\beta 1$ and $\beta 2$, we can see that the Log-Normal and Gamma are represented by a line. The Gaussian distribution is represented by a point and the Beta distribution is represented by an area (El Zaart and Ziou, 2007). From Fig. 1, we can conclude that Beta is the best distribution for modeling data in image. It is the best distribution since it can be used to approximate any shape of histogram (Gonzalez and Woods, 2008).

For those reasons we have chosen to apply the Beta distribution in our method. In the following we will explain in details the Beta distribution.

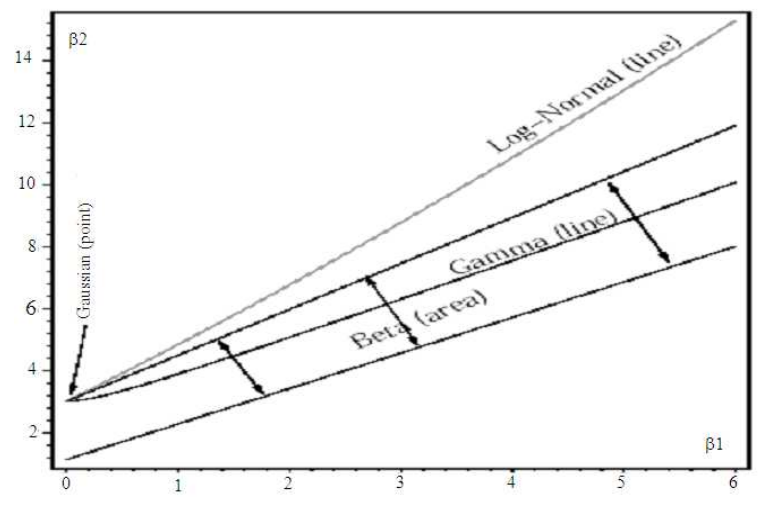

Fig. 1: Gaussian, Gamma, Beta and log-normal distributions (El Zaart and Ziou, 2007)

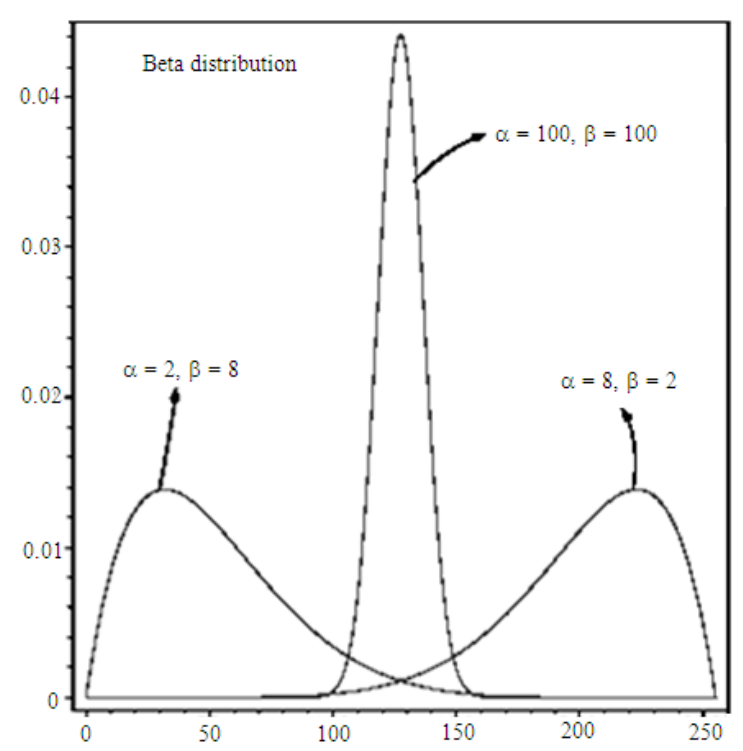

Fig. 2: Three Beta distributions with their parameters (El Zaart and Ziou, 2007)
Beta distribution: Beta distribution is a continuous probability distribution with the probability density function defined on the interval $[0,1]$ :

$$
f(x, \alpha, \beta)=\frac{\Gamma(\alpha+\beta)}{\Gamma(\alpha) \Gamma(\beta)} x^{\alpha-1}(1-x)^{\beta-1}
$$

Where:

$\alpha$ and $\beta=$ The shape parameters of the distribution and must be greater than zero

$\mathrm{x}=$ The intensity of the pixel and it must be between 0 and 1

$\Gamma \quad=$ The gamma function

The Beta distribution can take different shapes depending on the values of the two parameters (Fig. 2).

If $\alpha=\beta$ then the Beta distribution is symmetric, in Fig. 2 for $\alpha=\beta=100$. If $\alpha<\beta$ then the Beta distribution is skewed to right, Fig. 2 for $\alpha=2$ and $\beta=8$. If $\alpha>\beta$ then the Beta distribution is skewed to the left, in Fig. 2 for $\alpha=8$ and $\beta=2$. The moment-based estimators for $\alpha$ and $\beta$ and are given by (Evans et al., 2000; Jambunathan, 1954):

$$
\begin{gathered}
\alpha=\hat{\mathrm{m}}_{1}\left(\hat{\mathrm{m}}_{1}-\hat{\mathrm{m}}_{2}\right) /\left(\left(\hat{\mathrm{m}}_{2}-\hat{\mathrm{m}}_{1}^{2}\right)\right) \\
\beta=\frac{\left(\hat{\mathrm{m}}_{1}-1\right)\left(\hat{\mathrm{m}}_{2}-\hat{\mathrm{m}}_{1}\right)}{\left(\hat{\mathrm{m}}_{2}-\hat{\mathrm{m}}_{1}^{2}\right)}
\end{gathered}
$$

where, the $\mathrm{r}^{\text {th }}$ sample moment of a homogenous region $\left(\mathrm{R}_{\mathrm{k}}\right)$ is given by:

$$
\hat{\mathrm{m}}_{\mathrm{r}}=\left(\sum_{\mathrm{i} \in \mathrm{R}_{\mathrm{K}}} \mathrm{h}\left(\mathrm{x}_{\mathrm{i}}\right) \mathrm{x}_{\mathrm{i}}^{\mathrm{r}}\right) / \sum_{\mathrm{i} \in \mathrm{R}_{\mathrm{K}}} \mathrm{h}\left(\mathrm{x}_{\mathrm{i}}\right)
$$

We assume that an histogram skin images is formed by a mixture of Beta distributions:

$$
h\left(x_{j}\right)=\sum_{i=1}^{M} f\left(x_{j}, \alpha_{i}, \beta_{i}\right) p_{i}
$$

Where:

$\mathrm{M} \quad=$ The number of modes (classes) of the skin image histogram (skin image)

$\left(\alpha_{i}, \beta_{i}, p_{i}\right)=$ The statistical parameter of the $i^{\text {th }}$ mode of the skin image histogram

In this study, the objective is to estimate statistical parameters of a mixture of Beta distributions and use them to estimate the thresholds. The rest of the study is organized as follows: materials and methods, results, discussion and conclusion. 


\section{MATERIALS AND METHODS}

Proposed method: The unsupervised learning technique algorithm using a mixture of Beta distributions subdivides an image into a set of disjoint regions. Regions are then merged if either the number of members (pixel) in a region is less than a certain threshold (minimum class members input from user) or if the distance between the centers of two regions is less than a certain threshold (minimum class mean distance also input from the user). The following is the algorithm for the proposed method:

1. Input skin image.

2. Select a homogeneous test (standard deviation).

3. Calculate the image histogram.

4. Calculate the statistical parameters of Beta distributions and then estimate threshold value.

5. Split the image. This is an iterative process that splits any non-homogenous region until all regions are homogenous.

6. Merge any two homogeneous regions if merging criteria holds.

7. Output segmented image.

Next, we will explain the 5th and 6th steps.

Split image: For each class, apply a homogeneous test on it; if it is not homogeneous then split it by repeating the following steps from 1-6. The steps for splitting are:

1. Estimate initial threshold $\mathrm{T}^{0} ; \mathrm{T}^{0}=$ Average gray level value of the class to be spitted.

2. Split the class into two classes $\mathrm{C}_{1}$ and $\mathrm{C}_{2}$ according to $\mathrm{T}^{0}$.

3. Estimate values of $\alpha$ and $\beta$ for each class $\left(\mathrm{C}_{1}\right.$ and $\mathrm{C}_{2}$ ).

4. Calculate prior probabilities $\mathrm{p}_{1}$ for $\mathrm{C}_{1}$ and $\mathrm{p}_{2}$ for $\mathrm{C}_{2}$.

Let $\mathrm{n}_{1}$ and $\mathrm{n}_{2}$ be the number of pixels in class $\mathrm{C}_{1}$ and $\mathrm{C}_{2}$ respectively:

$$
\mathrm{n}_{1}=\sum_{\mathrm{i} \in \mathrm{C}_{1}} \mathrm{~h}\left(\mathrm{x}_{\mathrm{i}}\right) \text { and } \mathrm{n}_{2}=\sum_{\mathrm{i} \in \mathrm{C}_{2}} \mathrm{~h}\left(\mathrm{x}_{\mathrm{i}}\right)
$$

Thus:

$$
\mathrm{p}_{1}=\mathrm{n}_{1} /\left(\mathrm{n}_{1}+\mathrm{n}_{2}\right) \text { and } \mathrm{p}_{2}=\mathrm{n}_{2} /\left(\mathrm{n}_{1}+\mathrm{n}_{2}\right)
$$

5. Calculate the new threshold $\mathrm{T}^{\text {new }}$ by using statistics of both classes $C_{1}\left(\alpha_{1}, \beta_{1}, p_{1}\right)$ and $C_{2}\left(\alpha_{2}, \beta_{2}, p_{2}\right)(E l$ Zaart and Ziou, 2007):

$$
\mathrm{T}^{\text {new }}=1-\mathrm{e}^{\left(\mathrm{A}+\mathrm{B} \log \left(\mathrm{T}^{0}-1\right) / \mathrm{C}\right)}
$$

Where:

$$
\begin{aligned}
& \mathrm{A}=\log \left(\left(\mathrm{p}_{1} \mathrm{~K}_{1}\right) /\left(\mathrm{p}_{2} \mathrm{~K}_{2}\right)\right) \\
& \mathrm{B}=\alpha-\alpha_{2} \\
& \mathrm{C}=\beta_{1}-\beta_{2}
\end{aligned}
$$

Where:

$$
\mathrm{K}_{\mathrm{r}}=\frac{\Gamma\left(\alpha_{\mathrm{r}}+\beta_{\mathrm{r}}\right)}{\Gamma\left(\alpha_{\mathrm{r}}\right) \Gamma\left(\beta_{\mathrm{r}}\right)}
$$

In this step, estimation of threshold is an iterative process. The initial value:

$$
\mathrm{T}^{0}=\left(\mu_{1}+\mu_{2}\right) / 2
$$

where, $\mu_{\mathrm{r}}=\alpha_{\mathrm{r}} /\left(\alpha_{\mathrm{r}}+\beta_{\mathrm{r}}\right)$.

6. If $\left|\mathrm{T}^{0}-\mathrm{T}^{\text {new }}\right|>1$ then $\mathrm{T}^{0}=\mathrm{T}^{\text {new }}$ and repeat step 2 until step 5. Else record $\mathrm{T}^{\text {new }}$ in thresholds list and record two classes $\mathrm{C} 1$ and $\mathrm{C} 2$ where pixels in $\mathrm{C} 1$ are less than or equal to $\mathrm{T}^{\text {new }}$ and pixels in $\mathrm{C} 2$ are greater than $\mathrm{T}^{\text {new }}$.

Merge regions/classes: After splitting all nonhomogeneous classes in image, a merging step is necessary to merge some wrong classes that are produced from splitting step. The fundamental steps of merging include the following:

- If number of pixels in class is less than minimum class members then merge this class with the nearest class to it i.e., the class who has the closest mean.

- If the distance between the mean of a class and the mean of another class is less than a certain minimum class mean distance then, the two classes are merged.

- The result new mean will be the average of the two merged classes.

- The threshold between these two classes will be removed from the list of thresholds. Both of the minimum class members and minimum class mean distance are user input.

\section{RESULTS}

The proposed method of skin images segmentation was implemented and tested on different skin images. In this study, we present only three skin images. We 
compared our result with the result of unsupervised learning technique with Gamma (El-Zaart, 2010). We consider the first skin cancer image presented in Fig. 3. Figure 3a represents the original image. Table 1 presents the numerical results of applying our method on Fig. 3a. Figure $3 \mathrm{~b}$ represents the segmented image using the unsupervised learning technique with Gamma distribution (El-Zaart, 2010). Figure 3c, represents the segmented image using the unsupervised learning technique with Beta distribution (proposed method). Figure $3 \mathrm{~d}$ represents the original image with the interested object, which is the skin cancer region. By doing a comparison for this result, we can find that the method used Beta is better than the method used Gamma.

We consider a second skin cancer image presented in Fig. 4. Figure 4a represents the original image. Figure $4 \mathrm{~b}$ represents the segmented image using the unsupervised learning technique with Gamma distribution (El-Zaart, 2010). Figure 4c, represents the segmented image using the unsupervised learning technique with beta distribution (proposed method).

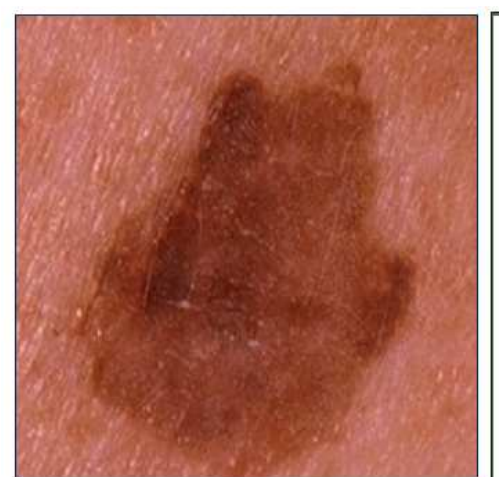

(a)

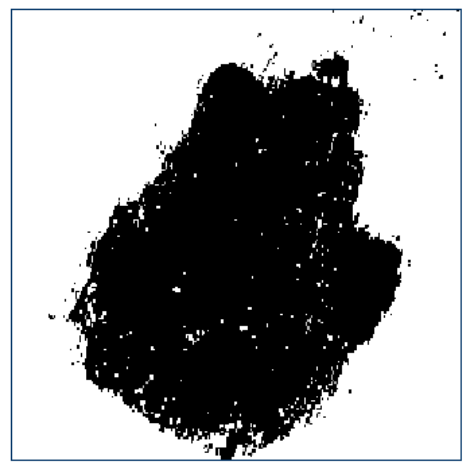

(c)

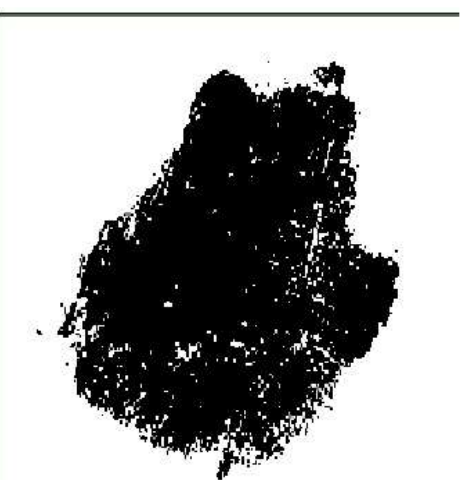

(b)

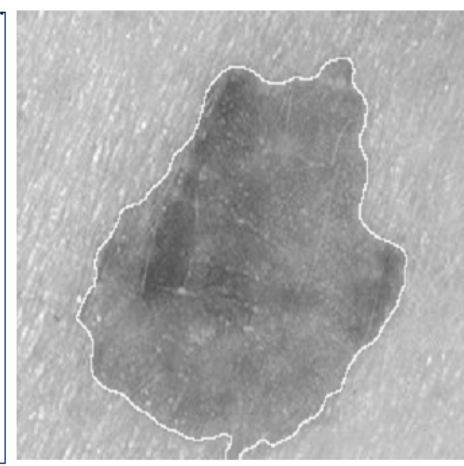

(d)

Fig. 3: (a) original image; (b) segmented image with Gamma (El-Zaart, 2010); (c) segmented with Beta (our method); (d) skin caner region defined by boundaries using Beta result

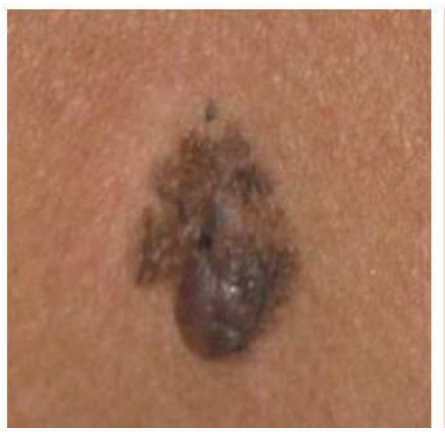

(a)

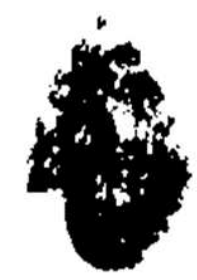

(b) 


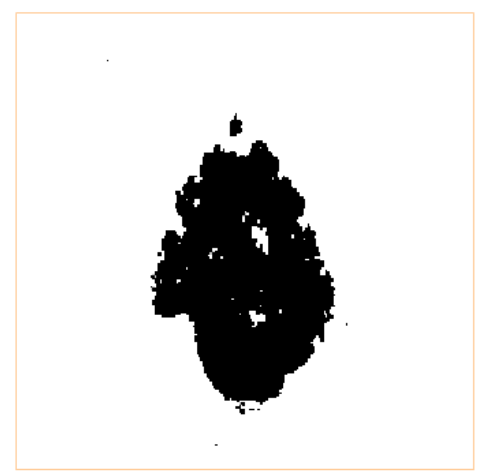

(c)

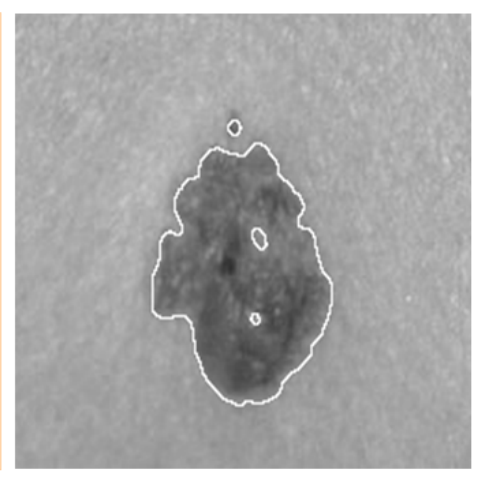

(d)

Fig. 4: (a) Original image; (b) segmented image with Gamma (El-Zaart, 2010); (c) segmented with Beta (our method); (d) skin caner region defined by boundaries using Beta result

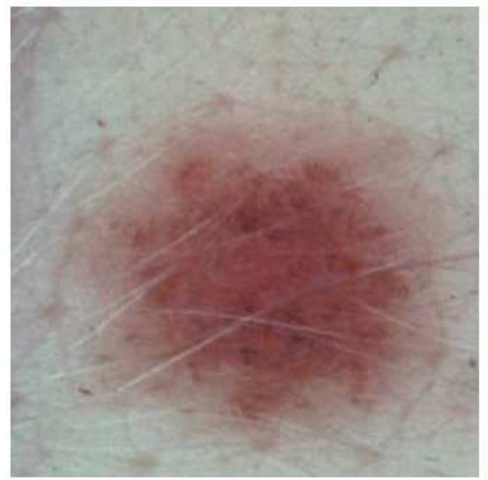

(a)

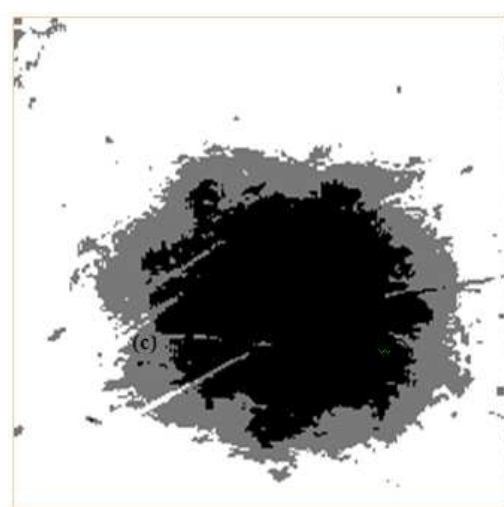

(c)

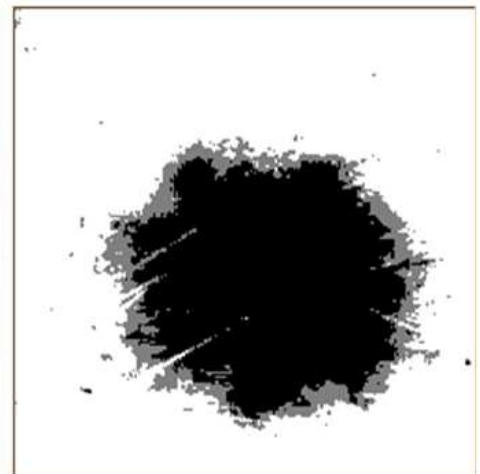

(b)

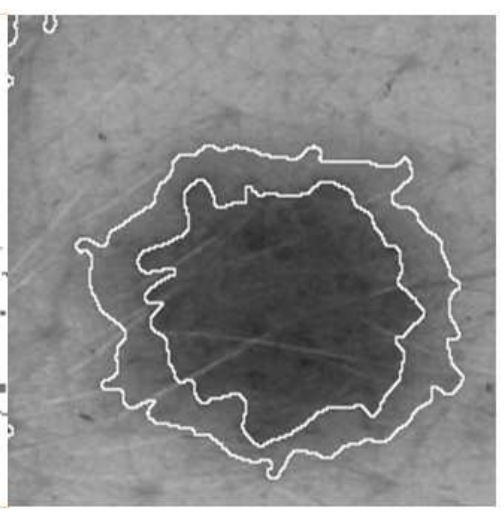

(d)

Fig. 5: (a) Original image; (b) segmented image with Gamma (El-Zaart, 2010); (c) segmented with Beta (our method); (d) skin caner region defined by boundaries using Beta result

Table 2 represents the numerical results of our method. Figure $4 \mathrm{~d}$ represents the original image with the interested object, which is the skin cancer region. By doing a comparison for this result, we can find that the method used Beta is better than the method used Gamma.
Table 1: Numerical results for image presented in Fig. 3a Threshold $=85$ and $\mathrm{M}=2$

Estimated parameters

First mode (class)

Second modes (class) $\beta$ 62.97 
Table 2: Numerical results for image presented in Fig. 4a

\begin{tabular}{llll}
\hline Threshold $=86$ and $\mathrm{M}=2$ & & & \\
Estimated parameters & $\mathrm{p}$ & $\alpha$ & $\beta$ \\
\hline First mode (class) & 0.15 & 21.69 & 67.43 \\
Second modes (class) & 0.85 & 110.7 & 152.2 \\
\hline
\end{tabular}

Table 3: Numerical results for image presented in Fig. 5a

$\mathrm{T} 1=87, \mathrm{~T} 2=121$ and $\mathrm{M}=3$

\begin{tabular}{llll} 
Estimated parameters & $\mathrm{p}$ & $\alpha$ & $\beta$ \\
\hline First mode (class) & 0.21 & 46.38 & 123.2 \\
Second modes (class) & 0.18 & 63.76 & 62.97 \\
Third mode (class) & 0.61 & 68.89 & 51.31 \\
\hline
\end{tabular}

We consider a third skin cancer image presented in Fig. 5. Figure 5a represents the original image. Figure $5 \mathrm{~b}$ represents the segmented image using the unsupervised learning technique with Gamma distribution (El-Zaart, 2010). Figure 5c, represents the segmented image using the unsupervised learning technique with beta distribution (proposed method).

Table 3 represents the numerical results of our method. Figure $5 \mathrm{~d}$ represents the original image with the interested objects, which are the skin cancer regions. By doing a comparison for this result, we can find that the method used Beta is better that the method used Gamma.

\section{DISCUSSION}

From Fig. 3-5, we can remark the following points:

- The unsupervised learning technique with Gamma gave poor results in segmentation of skin images. In Gamma result, the cancer region is smaller than the cancer region in Beta result

- In the case of three classes, Gamma result classified the region between cancer and noncancer skin as small region. However, Beta result segmented well this region (Fig. 5d)

- The results obtained by our proposed method is based on the user input. Our method requires an estimation of minimum class members and minimum class mean distance in order to improve the quality of segmentation

\section{CONCLUSION}

Image segmentation is a major task of image processing. It is an important technique used to identify related objects in an image. Many methods exist for image segmentation that attempts to segment an image into homogenous regions. Among those methods are classification-based methods that were used in this study. In this study, we proposed a new method that uses a unsupervised learning technique with Beta distribution. Because Beta represents almost any shape of an image histogram, the Beta distribution was chosen for parameter estimation in the proposed method. Experimental results showed good segmentation of skin images. The results obtained by our proposed method is based on minimum class members and minimum class mean distance which are input by the user. As future work, we will develop a method that estimates these two user inputs.

\section{ACKNOWLEDGMENT}

I would like to thank Amany Al-Saleh for her help in preparation of this paper. This work was supported by the Research Center of the College of Computer and Information Sciences, King Saud University, Riyadh, Saudi Arabia.

\section{REFERENCES}

El Zaart, A., D. Ziou, S. Wang and Q. Jiang, 2002. Segmentation of SAR images. Patt. Recog., 35: 713-724. DOI: 10.1016/s0031-3201(01) 00070-x

El Zaart, A., A. Al-Mejrad and A. Saad, 2004. Segmentation of mammography images for breast cancer detection. Proceedings of the Kuala Lumpur International Conference on Biomedical Engineering, (BE'04), Department of Biomedical Engineering, Malaysia, pp: 225-228.

El Zaart, A. and D. Ziou, 2007. Statistical modeling of multimodal SAR images. Int. J. Remote Sens., 28: 2277-2294. DOI: 10.1080/01431160600933997

El-Zaart, A., 2010. Image thresholding using ISODATA technique with Gamma distribution. J. Patt. Recog. Image Anal., 20: 29-41. DOI: 10.1134/S1054661810010037

Ercal, F., M. Moganti, W.V. Stoecker and R.H. Moss, 1993. Detection of skin tumor boundaries in color images. IEEE Trans. Med. Imag., 12: 624-626. DOI: $10.1109 / 42.241892$

Evans, M., N. Hastings and B. Peacock, 2000. Statistical Distribution. 3rd Edn., WileyInterScience, New York, ISBN: 10: 0471371246, pp: 34-42.

Ferrari, R.J., R.M., R.A. Borges and A.F. Frere, 2004. Segmentation of the fibro-glandular disc in mammograms using Gaussian mixture modeling. Med. Biologic. Eng. Comput., 42: 378-387. DOI: 10.1007/BF02344714

Gonzalez, R.C. and R.E. Woods, 2008. Digital Image Processing. 3rd Edn., Prentice-Hall, Inc., New Jersey, ISBN: 10: 013168728x, pp: 594. 
Jambunathan, M.V., 1954. Some properties of beta and gamma distributions. Ann. Math. Stat., 25: 401-405. DOI:10.1214/aoms/1177728800

Mitsias, P.D., M.A. Jacobs, R. Hammond, M. Pasnoor, S. Santhakumar and N.I. Papamitsakis et al., 2002. Multiparametric MRI ISODATA ischemic lesion analysis: Correlation with the clinical neurological deficit and single-parameter MRI techniques. J. Am. Heart Assoc., 33: 2839-2844. DOI: 10.1161/01.STR.0000043072.76353.7C
Xua, L., M. Jackowski, A. Goshtasby, D. Roseman and S. Bines et al., 1999. Segmentation of skin cancer images. Image Vis. Comput., 17: 65-74. DOI: 10.1016/S0262-8856(98)00091-2 